\title{
An Analytical Study for Two-Hop Amplify-and-Forward DS-CDMA Systems over Rayleigh/Nakagami-m Fading Channels
}

\author{
Nuri Kapucu, Mehmet Bilim, Ibrahim Develi \\ Department of Electrical \& Electronics Engineering, Faculty of Engineering, \\ Erciyes University, 38039, Kayseri, Turkey \\ nurikapucu@erciyes.edu.tr; bilim@erciyes.edu.tr; develi@erciyes.edu.tr
}

\begin{abstract}
This paper presents analytical solutions for the performance of a single relay aided amplify-and-forward (AF) direct sequence code division multiple access (DS-CDMA) system over Rayleigh/Nakagami- $m$ fading channels. The source-destination channel is assumed to be Rayleigh distributed while relay-destination link is Nakagami- $m$ distributed. First, a cumulative distribution function (CDF) is derived for the source-relay-destination link and the derived CDF expression is used to evaluate the outage probability. Then, a moment generating function (MGF) is obtained through integral evaluation of the derived CDF expression in order to evaluate the average error probability of the considered system. Finally, the accuracy of the proposed analytical expressions are confirmed by computer simulations.
\end{abstract}

Keywords: Fixed gain relaying, DS-CDMA, Cumulative distribution function, Moment generating function.

\section{Introduction}

Relaying is one of the promising techniques to maintain the communication, extend the coverage range and achieve high transmission rates. In practice, it may be impossible to communicate from transmitter to receiver due to fading effects, path loss and shadowing. In these conditions, two-hop relaying can be used as an alternative transmission technique in practical systems such as CDMA systems or next generation wireless systems. There are two main relaying methods such as amplify-and-forward (AF) relaying and decode-and-forward (DF) relaying. In AF method, the received signal is amplified at relay $(R)$ node and the amplified source $(S)$ signal is sent to the destination $(D)$. On the other hand, $R$ sends the estimated signal to $D$ in DF relaying method [1].

In many publications, the performance of two-hop relaying systems is investigated [2]-[8]. The authors in [2, 3] presented a study on dual-hop fixed gain relaying systems over Rayleigh/Rician and Rician/Rayleigh fading channels. In these works, they derived a cumulative distribution function (CDF) to analyse the system performance. In [4, 5], the authors proposed exact expressions for two-hop AF relaying systems over Nakagami- $m /$ Rician fading channels as well as Rician/Rician and Nakagami- $m /$ Nakagami- $m$ fading channels. Kapucu et al. [6, 7] investigated the performance of two-hop regenerative relaying systems under mixed Rayleigh/Gamma and Rayleigh/Weibull fading conditions. In [8], the end-toend performance of fixed gain two-hop AF relaying is presented in flat Rayleigh fading.

In the studies above [2]-[8], two-hop relaying systems are investigated over different fading channels without DSCDMA systems. DS-CDMA system is a spread spectrum technique which is commonly used in mobile communication systems. In DS-CDMA systems, the main objective is to increase bandwidth and provide a safe communication in a limited frequency band [9]-[11]. In the literature, there are only a few studies dealing with the performance of two-hop DS-CDMA networks with AF transmission [12]-[14]. The bit-error rate performance of two-hop AF relaying with DS-CDMA systems is investigated over different fading channels by presenting only computer simulations. In [13], the authors analysed the performance of DS-CDMA system with relaying by taking into account multi-user transmitter pre-processing based on channel impulse responses. Zappone et al. [14] considered the resource allocation problem in a relay-aided DS-CDMA wireless communication system.

In this study, different from [2]-[14], the performance of two-hop DS-CDMA systems with AF relays is presented over mixed fading channels where the $S-R$ and $R-D$ links are subject to Rayleigh and Nakagami- $m$ fading, respectively. For the considered system, the main contributions of this paper is explained as follows 
1) An exact solution for the CDF of indirect link ( $S-R-D$ link) is derived for fixed gain AF relaying based two-hop DS-CDMA systems and the outage probability of the considered system is evaluated based on the derived CDF expression.

2) An exact solution for the moment generating function (MGF) is derived by using the exact CDF expression and average error probability is examined based on MGF approach.

3) The numerical results are confirmed by the computer simulations to show the accuracy of the derived expressions.

\section{System and Channel Models}

Consider an $\mathrm{N}$-user DS-CDMA network with fixed gain AF relaying protocol in asymmetric fading environments. A single-relay two-hop relaying system is illustrated in Fig.1. In the considered system, each user is equipped with one antenna and half duplex transmission is assumed. The transmission occurs in two transmission phases. In the first phase, the $S$ forwards the message to the $R$ node.

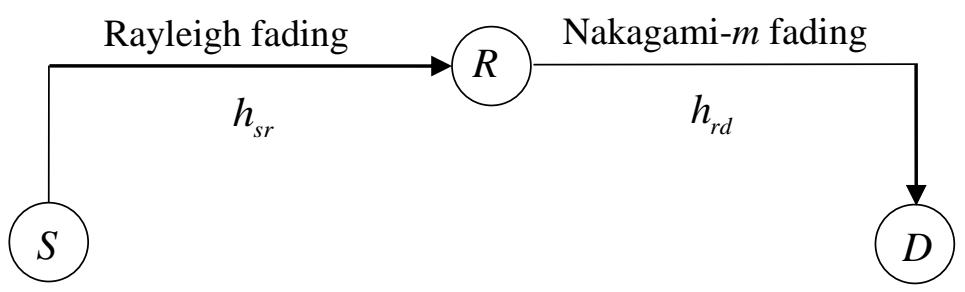

Fig. 1: Illustration of single-relay two-hop system model.

The received DS-CDMA signal at $R$ node can be expressed as

$$
r_{s r}(t)=\sum_{i=0}^{f-1} \sum_{n=1, n \neq r}^{N} x_{n}(i) C_{n}\left(t-\tau_{n}-i T_{b}\right) h_{s r}(i)+n_{s r}(t)
$$

where $f$ is the frame length, $N$ is the number of users, $x_{n}(i)$ and $C_{n}$ are the information signal and the spreading code for the $n$th user, respectively. $T_{b}$ is the bit period and $\tau_{n}$ represents transmission delay of the $n$th user. $n_{s r}(t)$ is the additive white Gaussian noises (AWGN) and $h_{s r}$ is the Rayleigh fading coefficient for the $S-R$ path. The PDF of $S-R$ path for the instantaneous SNR can be given according to Rayleigh distribution by

$$
f_{\gamma_{s r}}\left(\gamma_{s r}\right)=\frac{1}{\bar{\gamma}_{s r}} \exp \left(-\frac{\gamma_{s r}}{\bar{\gamma}_{s r}}\right), \gamma_{s r} \geq 0
$$

where $\gamma_{s r}=h_{s r}^{2} E_{b} / N_{0}$ and $\bar{\gamma}_{s r}=\mathrm{E}\left(h_{s r}^{2}\right) E_{b} / N_{0}$ are the instantaneous and average SNRs, respectively. $E_{b}$ is the energy per bit and $\mathrm{E}(\cdot)$ denotes the expectation operator.

In the second phase, $R$ node transmits the amplified information signal to the $D$ node. The amplified information signal at $D$ node is expressed as,

$$
r_{r d}(t)=\sum_{i=0}^{f-1} \sum_{n=1}^{N} r_{s r}\left(t-D_{n}-\tau_{r}-i l T_{b}\right) h_{r d}(i) \beta+n_{r d}(t)
$$


where $\beta=\sqrt{E_{1} /\left(\left(E_{0}\left|h_{s r}\right|^{2}\right)+N_{0}\right)}$ is the amplification factor, $E_{0}$ is the source energy and $E_{1}$ is the relay energy, $D_{n}$ is the time delay in the second transmission phase, $\tau_{r}$ is the transmission delay of $R$, and $n_{r d}(t)$ is AWGN of the $R-D$ link. $h_{r d}$ is the Nakagami- $m$ fading coefficient of $R-D$ link and the PDF of Nakagami- $m$ distributed $R-D$ link is given as follows

$$
f_{\gamma_{r d}}\left(\gamma_{r d}\right)=\frac{m \gamma_{r d}^{m-1}}{\bar{\gamma}_{r d}^{m} \Gamma(m)} \exp \left(-\frac{m \gamma_{r d}}{\bar{\gamma}_{r d}}\right), \gamma_{r d} \geq 0
$$

where $m$ is the fading parameter and $\Gamma(\cdot)$ is Gamma function. $\gamma_{r d}=h_{r d}^{2} E_{b} / N_{0}$ and $\bar{\gamma}_{r d}=\mathrm{E}\left(h_{r d}^{2}\right) E_{b} / N_{0}$ are the instantaneous and average SNRs, respectively.

\section{Statistical Analysis}

In this section, the CDF and MGF expressions are obtained in order to analyse system performance. The total instantaneous SNR of the considered system at $D$ node with fixed gain two-hop AF relaying is given by [8]

$$
\gamma_{s r d}=\frac{\gamma_{s r} \gamma_{r d}}{C+\gamma_{r d}}
$$

where $C \geq 0$.

\subsection{Outage Probability}

Outage probability, $P_{\text {out }}$, is defined as the probability of instantaneous total SNR, $\gamma_{\text {srd }}$, that falls below a threshold, $\gamma_{t h}$ where $\gamma_{t h}$ depends on the number of users, spread length, spectral efficiency and the cross-correlation matrix defined by

$$
\gamma_{t h}=\left(2^{2 S \Re / N}-1\right) /\left(2 S / N^{2}\left[R_{d}^{-1}\right]_{s, S}\right)
$$

where $\mathfrak{R}$ is the spectral efficiency, $\left\lfloor R_{d}^{-1}\right\rfloor_{s, s}$ denotes the sth row and the $s$ th column elements of the inverse of the cross-correlation matrix at the destination. $P_{\text {out }}$ is obtained according to

$$
P_{o u t}=F_{\gamma_{s d}}\left(\gamma_{t h}\right)=\operatorname{Pr}\left\lfloor\frac{\gamma_{s r} \gamma_{r d}}{C+\gamma_{r d}}<\gamma_{t h}\right\rfloor
$$

where $F_{\gamma_{s d}}\left(\gamma_{t h}\right)$ is the CDF of the total instantaneous SNR. $P_{\text {out }}$ can be given conditioned on the second hop SNR as

$$
P_{o u t}=\int_{0}^{\infty} \operatorname{Pr}\left[\gamma_{s r}<\frac{\gamma_{t h}\left(C+\gamma_{r d}\right)}{\gamma_{r d}} \mid \gamma_{r d}\right] f_{\gamma_{r d}}\left(\gamma_{r d}\right) d \gamma_{r d}
$$

In order to solve the integral in (8), we need to insert CDF expression of Rayleigh fading and the PDF of Nakagami$m$ fading into (8). The CDF of Rayleigh fading is expressed by 


$$
F_{\gamma_{s r}}\left(\gamma_{t h}\right)=1-\exp \left(-\frac{\gamma_{t h}}{\bar{\gamma}_{s r}}\right)
$$

By substituting (4) and (9) into (8), $P_{\text {out }}$ can be re-expressed as

$$
P_{\text {out }}=\int_{0}^{\infty}\left[1-\exp \left(-\frac{\gamma_{t h}\left(C+\gamma_{r d}\right)}{\bar{\gamma}_{s r} \gamma_{r d}}\right) \mid \frac{m_{r d}^{m_{r d}} \gamma_{r d}^{m_{r d}-1}}{\bar{\gamma}_{r d}^{m_{r d}} \Gamma\left(m_{r d}\right)} \exp \left(-\frac{m_{r d} \gamma_{r d}}{\bar{\gamma}_{r d}}\right) d \gamma_{r d}\right.
$$

After some mathematical arrangements, equation (10) can be rewritten as

$$
\begin{aligned}
P_{o u t} & =\int_{0}^{\infty} \frac{m_{r d}^{m_{r d}} \gamma_{r d}^{m_{r d}-1}}{\bar{\gamma}_{r d}^{m_{r d}} \Gamma\left(m_{r d}\right)} \exp \left(-\frac{m_{r d} \gamma_{r d}}{\bar{\gamma}_{r d}}\right) d \gamma_{r d} \\
& -\int_{0}^{\infty} \exp \left(-\frac{\gamma_{t h}}{\bar{\gamma}_{s r}}\left(1+\frac{C}{\gamma_{r d}}\right)\right) \frac{m_{r d}^{m_{r d}} \gamma_{r d}^{m_{r d}-1}}{\bar{\gamma}_{r d}^{m_{r d}} \Gamma\left(m_{r d}\right)} \exp \left(-\frac{m_{r d} \gamma_{r d}}{\bar{\gamma}_{r d}}\right) d \gamma_{r d}
\end{aligned}
$$

The closed-form solution of the integral in (11) is obtained with the help of [15, 3.351.3 and 3.471.9] and $P_{\text {out }}$ is derived as follows

$$
P_{\text {out }}=F_{\gamma_{s d}}\left(\gamma_{t h}\right)=\frac{(m-1) !}{\Gamma(m)}-\left(\exp \left(-\frac{\gamma_{t h}}{\bar{\gamma}_{s r}}\right) \frac{2 m^{m}}{\bar{\gamma}_{r d}^{m} \Gamma(m)}\left(\frac{\beta}{\alpha}\right)^{m / 2} K_{m}(2 \sqrt{\beta \alpha})\right)
$$

where $\beta=\left(C \gamma_{t h}\right) / \bar{\gamma}_{s r}, \alpha=m / \bar{\gamma}_{r d}$ and $K_{v}(\cdot)$ is the $v$ th-order modified Bessel function of the second kind.

\subsection{Average Error Probability}

Integral form of the average error probability for $M$-ary phase shift keying ( $M$-PSK) is given as

$$
P_{\text {error }}=\frac{1}{\pi} \int_{0}^{\theta} M_{\gamma_{s d d}}\left(\frac{g_{p}}{\sin ^{2} \theta}\right) d \theta
$$

where $g_{p}=\sin ^{2}(\pi / M)$ and $\theta=\frac{\pi(M-1)}{M}$. In order to evaluate the average error probability, it is required to find the MGF of the total SNR. MGF expression can be found through integral evaluation of the CDF expression derived in this work. The MGF expression can be obtained by solving the integral in (13).

$$
M_{\gamma_{s d d}}(s)=1-s \int_{0}^{\infty} e^{-s r}\left(1-F_{\gamma_{s d d}}(r)\right) d r
$$

By substituting (12) into (14), we have

$$
M_{\gamma_{s c d}}(s)=1-s \int_{0}^{\infty} e^{-s r}\left(1-\left(\left(\frac{(m-1) !}{\Gamma(m)}\right)-\left(\exp \left(-\frac{r}{\bar{\gamma}_{s r}}\right) \frac{2 m^{m}}{\bar{\gamma}_{r d}^{m} \Gamma(m)}\left(\frac{\beta}{\alpha}\right)^{m / 2} K_{m}(2 \sqrt{\beta \alpha})\right)\right) d r\right.
$$


Equation (15) is rearranged as

$$
\begin{aligned}
M_{\gamma_{s c d}}(s)=1-s\left\{\int_{0}^{\infty} e^{-s r} d r-\int_{0}^{\infty} e^{-s r}\left(\frac{(m-1) !}{\Gamma(m)}\right) d r\right. \\
\left.\quad+\int_{0}^{\infty} e^{-s r}\left(\exp \left(-\frac{r}{\bar{\gamma}_{s r}}\right) \frac{2 m^{m}}{\bar{\gamma}_{r d}^{m} \Gamma(m)}\left(\frac{\beta}{\alpha}\right)^{m / 2} K_{m}(2 \sqrt{\beta \alpha})\right) d r\right\}
\end{aligned}
$$

After solving the first and the second integral in (16), we have

$$
\begin{aligned}
M_{\gamma_{s d d}}(s) & =\frac{(m-1) !}{\Gamma(m)}-\left\{s \frac{2 m^{m}}{\bar{\gamma}_{r d}^{m} \Gamma(m)} \int_{0}^{\infty} e^{-s r} \exp \left(-\frac{r}{\bar{\gamma}_{s r}}\right)\left(\frac{(C r) / \bar{\gamma}_{s r}}{m / \bar{\gamma}_{r d}}\right)^{m / 2}\right. \\
& \left.\times K_{m}\left(2 \sqrt{\frac{C r m}{\bar{\gamma}_{s r} \bar{\gamma}_{r d}}}\right) d r\right\}
\end{aligned}
$$

With the help of $[15,6.643 .3]$, the MGF expression is derived as

$$
\begin{aligned}
M_{\gamma_{s d}}(s) & =\frac{(m-1) !}{\Gamma(m)}-\left\{s \frac{2 m^{m}}{\bar{\gamma}_{r d}^{m} \Gamma(m)}\left(\frac{C \bar{\gamma}_{r d}}{m \bar{\gamma}_{s r}}\right)^{m / 2} \frac{\Gamma(m+1) \Gamma(1)}{2 \sqrt{(m C) /\left(\bar{\gamma}_{s r} \bar{\gamma}_{r d}\right)}}\right. \\
& \times \exp \left(\left(\frac{m C}{\bar{\gamma}_{s r} \bar{\gamma}_{r d}}\right) / 2\left(\frac{1}{\bar{\gamma}_{s r}}+s\right)\right)\left(\frac{1}{\bar{\gamma}_{s r}}+s\right)^{-\left(\frac{m+1}{2}\right)} \\
& \left.\times W_{-\left(\frac{m+1}{2}\right) \cdot\left(\frac{m}{2}\right)}\left(\left(\frac{m C}{\bar{\gamma}_{s r} \bar{\gamma}_{r d}}\right) /\left(\frac{1}{\bar{\gamma}_{s r}}+s\right)\right)\right\}
\end{aligned}
$$

where $W_{\lambda, \mu}(\cdot)$ is the Whittaker function [15]. By substituting (18) into (13), average error probability is numerically obtained.

\section{Numerical Results}

This section presents the numerical results and their corresponding simulation results for outage probability and average error probability in Figs. 2 and 3, respectively. The results are plotted in terms of outage probability and average error probability as a function of average SNR $\left(\bar{\gamma}_{s r}=\bar{\gamma}_{r d}=\bar{\gamma}\right)$. The number of users is $N=4$ and the spectral efficiency is $\Re=1 \mathrm{bit} / \mathrm{s} / \mathrm{Hz}$ in all numerical results and simulation results.

Fig. 2 illustrates the outage performance of DS-CDMA systems with two-hop AF relaying for $m=1, S=2 N$, $m=3, S=N$ and $m=3, S=2 N$ over Rayleigh/Nakagami- $m$ fading channels. It can be observed that the numerical results obtained by the proposed exact expression of (12) and the simulation results are in well agreement, especially at medium and high SNR region (after $\approx 10 \mathrm{~dB}$ ). When $S=2 N$, the $\gamma_{t h}$ is greater than the case of $S=N$ (This case can be observed from equation (6)). So that, in the case of $m=1$ and $S=2 N$, the outage performance is the worst due to the greater value of $\gamma_{t h}$, as expected. In the case of $m=3, P_{\text {out }}$ equals $10^{-2}$ at $\approx 22 \mathrm{~dB}$ and $\approx 26 \mathrm{~dB}$ with $S=N$ and $S=2 N$, respectively. From this example, we can see that decreasing $S$ from $2 N$ to $N$ brings approximately $4 \mathrm{~dB}$ SNR gain. Moreover, it can be noted that the outage probability decreases by increasing the value of fading parameter (from $m=1$ to $m=3$ ) in the whole 
SNR region, as expected. For example, it can be seen that moving from $m=1$ to $m=3$ improves the outage probability of the system by approximately $7 \mathrm{~dB}$ when $P_{\text {out }}$ equals to $10^{-2}$.

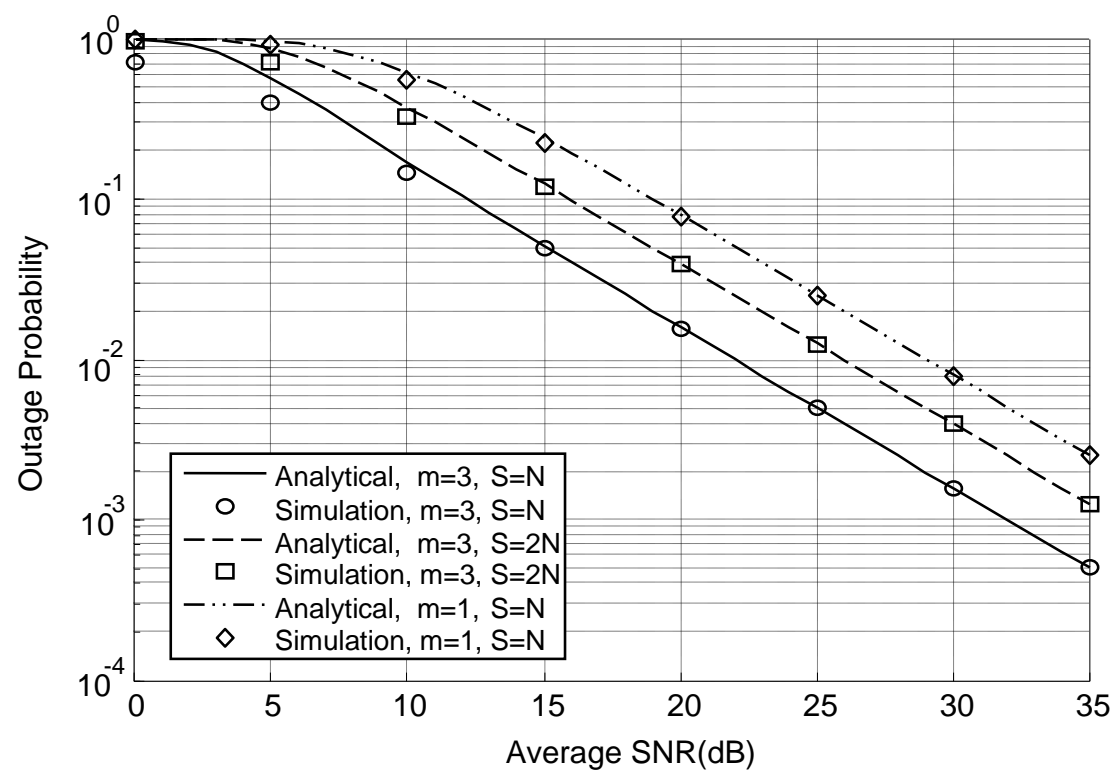

Fig. 2: Outage probability of two-hop AF relaying based DS-CDMA systems for different values of $m$.

Fig. 3 compares the error probability of two-hop DS-CDMA systems with AF relaying for different constellation sizes such as BPSK, QPSK and 16PSK modulations through integral evaluation of the derived MGF expression. In this example, the value of Nakagami- $m$ fading parameter, $m$, is set to 2 .

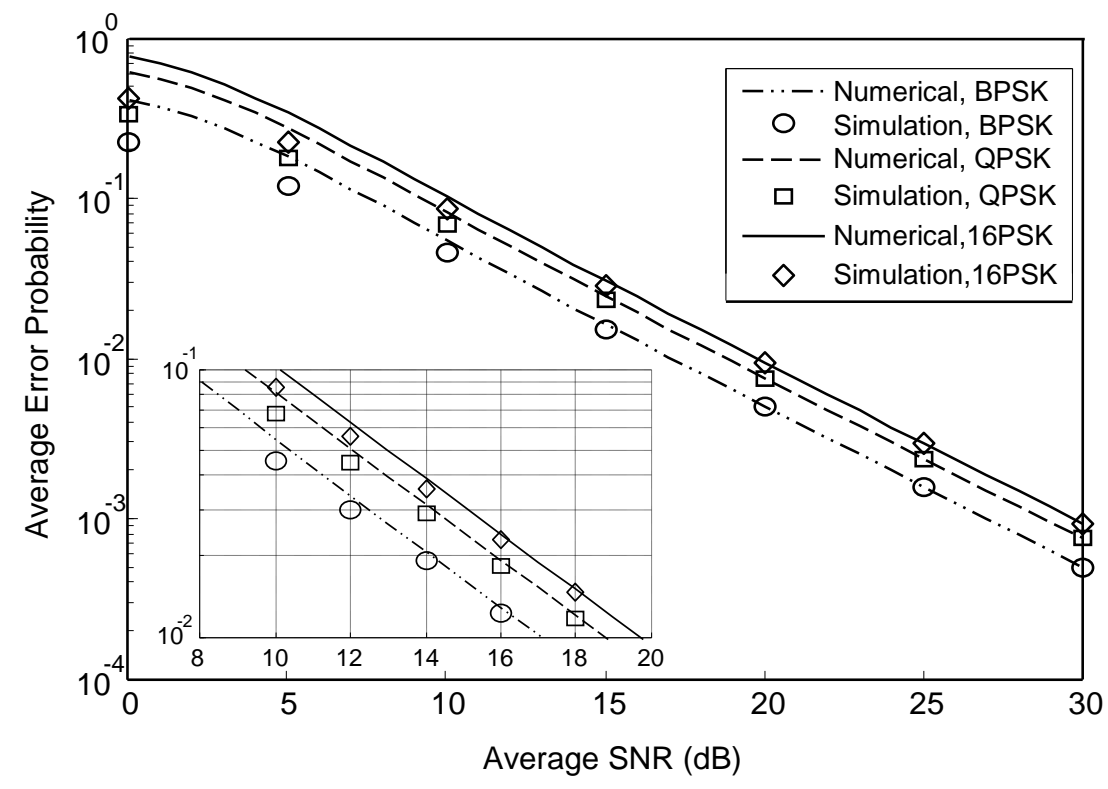

Fig. 3: Average error performance of two-hop AF relaying based DS-CDMA systems with for different constellation sizes of $M$-PSK modulations with $m=2$. 
Again, it is shown that the results of the error probability obtained by using the exact MGF expression of (18) derived in this work show excellent agreement with the simulation results especially after $\approx 12 \mathrm{~dB}$. As expected, when the constellation size gets larger, the average error probability increases. For example, average error probabilities of $P_{\text {error }} \approx 1.6 \times 10^{-2}$ and $P_{\text {error }} \approx 3.1 \times 10^{-2}$ occurs at $\approx 15 \mathrm{~dB}$ for BPSK and 16 PSK modulations, respectively.

\section{Conclusion}

In this study, we introduced an accurate analytical framework for investigating the performance of two-hop DSCDMA systems with fixed gain AF relaying over Rayleigh/Nakagami- $m$ asymmetric fading channels. We derived an exact $\mathrm{CDF}$ and MGF expressions for the total SNR of cascaded link ( $S-R-D$ link) in order to analyse the average error probability and outage probability. The numerical results obtained by the proposed expressions are in excellent agreement with their corresponding simulation results.

\section{Acknowledgements}

This study is supported by the Scientific and Technological Research Council of Turkey (TUBITAK) under project grant 114E242 and the Scientific Research Projects Coordinating Office of Erciyes University (EU-BAP, Project No: FDK-2014-5166).

\section{References}

[1] J. N. Laneman, D. N. C. Tse and G. W. Wornell, "Cooperative diversity in wireless networks: Efficient protocols and outage behavior," IEEE Trans. Inf. Theory, vol. 50, no. 12, pp. 3062-3080, 2004.

[2] H. A. Suraweera, R. H. Y. Louie, Y. Li, G. K. Karagiannidis and B. Vucetic, "Two hop amplify-and-forward transmission in mixed Rayleigh and Rician fading channels," IEEE Commun. Lett., vol. 13, no. 4, pp. 227-229, 2009.

[3] H. A. Suraweera, G. K. Karagiannidis and P. J. Smith, "Performance analysis of the dual-hop asymmetric fading channel," IEEE Trans. Wireless Commun., vol. 8, no. 6, pp. 2783-2788, 2009.

[4] S. S. Soliman and N. C. Beaulieu, "Dual-hop AF relaying systems in mixed Nakagami-m and Rician links," in Proceedings of the IEEE GLOBECOM Workshops, Anaheim, CA, USA, 2012, pp. 447-452.

[5] S. S. Soliman and N. C. Beaulieu, "The bottleneck effect of Rician fading in dissimilar dual-hop AF relaying systems," IEEE Trans. Veh. Technol., vol. 63, no. 4, pp. 1957-1965, 2014.

[6] N. Kapucu, M. Bilim and I. Develi, "Outage probability analysis of dual-hop decode-and-forward relaying over mixed Rayleigh and generalized Gamma fading channels," Wireless Pers. Commun., vol. 71, no. 2, pp. 947-954, 2013.

[7] N. Kapucu, M. Bilim, I. Develi and Y. Kabalci, "Performance of two-hop relay assisted decode-and-forward transmission under mixed fading environments," Elektronika ir Elektrotechnika, vol. 21, no. 1, pp. 60-63, 2015.

[8] M. O. Hasna and M-S. Alouini, "A performace study of dual-hop transmissions with fixed gain relays," IEEE Trans. Wireless Commun., vol. 3, no. 6, pp. 1963-1968, 2004.

[9] S. Moshavi, "Multi-user detection for DS-CDMA communications," IEEE Communications Magazine, vol. 34, no. 10, pp. 124-136, 1996.

[10] C. Ciftlikli and I. Develi, "A simple and useful approach for the determination process of the weighted despreading sequences in a DS-CDMA system," European Trans. on Telecommunications, vol. 14, no. 4, pp. 361-366, 2003.

[11] I. Develi, "Detection of optimal spreading codes for DS-CDMA wireless systems with despreading sequences weighted by adjustable chip waveforms," Journal of the Franklin Institute, vol. 342, pp. 69-84, 2005.

[12] N. Kapucu, M. Bilim and I. Develi, "Performance of two-hop DS-CDMA systems using amplify-and-forward protocol over different fading channels," Elektronika ir Elektrotechnika, vol. 22, no. 1, pp. 361-366, pp. 97-101, 2016.

[13] N. V. A. Murali, P.Nagaradjane and S. P. Damodaran, "Performance of relay-aided downlink DS-CDMA system using transmitter preprocessing based on feedback information," Computers and Electrical Engineering, vol. 40, no. 4, pp. 1306-1315, 2014.

[14] A. Zappone and E. A. Jorswieck, "Resource allocation in amplify-and-forward relay-assisted DS/CDMA systems," IEEE Trans. Wireless Commun., vol. 11, no. 4, 2012.

[15] I. S. Gradshteyn and I. M. Ryzhik, Table of Integrals, Series and Products, New York: Academic, 2007. 